\title{
Impact of Gaming Disorder Against Adolescent Emotional Intelligence: A Systematic Review
}

\author{
${ }^{1}$ Ari Dwi Sulaksono, ${ }^{2}$ Wisnu Barlianto, ${ }^{3}$ Heni Dwi Windarwati \\ ${ }^{1}$ Student of Masters in Mental Health Nursing, Brawijaya University, Malang \\ Indonesia \\ ${ }^{2,3}$ Faculty of Medicine, Brawijaya University, Malang, Indonesia \\ Email: aridwisulaksono@gmail.com
}

\begin{abstract}
Emotional intelligence consists of self-awareness, self-regulation, self-motivation, selfempathy and social skills. Gaming disorder in adolescents can lead to self-concept disorder, depression, social dysfunction and even mental disorders. The systematic aim of this review is to identify the phenomenology of the impact of gaming disorder on adolescent emotional intelligence. The stages in making this systematic review create questions, identification, eligibility, article inclusion selection, screening, and appraisal in an international database that is Science Direct, ProQuest and PubMed. Selection was done with PRISMA flow-diagrams and criticized with the JBI tool. After obtaining 23 relevant articles, they are then analyzed into a systematic review. Gaming disorder is a problem of great concern in various countries. Gaming disorder is the main trigger affecting the emotional intelligence of adolescents today. The highest aspect resulting from gaming disorder is in the aspect of self-regulation, then aspects of self-awareness, self-empathy, self-motivation and social skills also experience a significant impact. This disorder, if it continues with higher intensity, can cause sufferers to experience negative things in their lives. Emotional intelligence is very important to be mastered in life, if emotional intelligence has been disturbed due to gaming disorder then the five aspects of emotional intelligence will also be disturbed. The fact that gaming disorder is empirically proven can have an impact on adolescent emotional intelligence. Thus, conducting additional research in Indonesia and also the world is very important to understand gaming disorder and how to handle it.
\end{abstract}

Keywords: Gaming Disorder, Internet Gaming Disorder, ICD-11, Emotional Intelligence, Gaming Behavior Adolescen.

\section{A. INTRODUCTION}

Rapid technological developments, especially in entertainment (games) can make teens addicted to playing games (Kurt et al., 2018; Paik et al., 2017; Wartberg, Zieglmeier, \& Kammerl, 2019). Gaming disorder is a pattern of negative behavior characterized by loss of control over a game (Saunders, 2017; Paik et al., 2017). The fifth edition of the American Psychiatric Association (APA) Diagnostic and Statistics Manual of Mental Disorders (DSM-5) has classified gaming disorder as a subset of non-substance-related disorders in the category of substance-related and addictive disorders (APA, 2013).

Diagnostic criteria for gaming disorder are: (a) preoccupation with the game, (b) withdrawal symptoms when the game is stopped, (c) increased tolerance for the need to spend more time involved in the game, (d) failed attempts to control participation in the game, (e) losing interest in hobbies and other entertainment as a result of the game, (f) continuing to use Internet games excessively despite being 
aware of psychosocial problems, (g) deceiving family members, therapists, or others regarding the amount of game use, $(\mathrm{h})$ using games to escape or eliminate negative moods and (i) losing significant relationships, jobs, or educational opportunities due to participating in games (APA, 2013; Wang et al., 2019; Milani et al., 2017; Kurt et al., 2018).

According to a recent epidemiological survey study gaming disorders experienced by adolescents can have physical, psychological and social impacts. Physical impacts include amblyopia on the eyes, and tendon injuries (Ayenigbara, 2018; Meng et al., 2015; Dong et al., 2017; Weinstein \& Lejoyeux, 2015; Marino \& Spada, 2017). Psychological effects result in decreased self-concept, depression, attention deficit hyperactivity disorder (ADHD), obsessive compulsive disorder (Chi, Lin, \& Zhang, 2016; Andreassen et al., 2016; Lemenager et al., 2018; Liau et al., 2015; Hawi, Samaha \& Griffiths, 2018; Satghare et al., 2016). The social impact of game addiction results in loss of learning time, family function, relationships with peers, difficulty expressing oneself and work (John B. Saunders, 2017; Mei et al., 2016; Wu et al., 2016; Gentile et al., 2017; Chi, Lin, \& Zhang, 2016; Samek, Hicks, Keyes et al., 2015). This shows that adolescents who are addicted to the game will experience problems of visual function, musculoskeletal, self-concept, impaired concentration and relationships between people.

Despite its serious impact on life, gaming disorder is still only a candidate for psychiatric diagnosis. The number of studies on gaming disorder treatment is quite limited in the literature, and effective and specific treatment protocols for this disorder have not yet been developed. To our knowledge, there is no comprehensive or empirical research in Indonesia on this topic. Thus, this study aims to systematically review studies published today that identify the impact of gaming disorder on adolescent emotional intelligence.

\section{B. METHOD}

The systematic review was carried out through several stages, namely making research questions according to PICO rules. Furthermore, collecting data for this literature review using diagrams includes: identification, screening, eligibility selection, and determination of inclusion criteria. In the final stage, a review is carried out by synthesizing the literature to obtain a systematic review.

\section{Research Questions}

The research question in this review is, "What is the Impact of Gaming Disorder on Adolescent Emotional Intelligence?

\section{Identification of Relevant Journals from Title / Abstract}

Article identification is done by searching journal articles in three databases. The database used by the author is Science Direct, ProQuest and PubMed. Then before conducting a search, the authors choose the settings on each database search page, namely: articles published between 2015-2019, the type of reference is the 
original international article. After completing the search settings then search for articles using keywords. The keywords used by the authors in this systematic review are 5 in each database, namely Gaming disorder, Internet gaming disorder, ICD-11, Emotional intelligence and Gaming behavior adolescent. The reference chosen for synthesis must meet the inclusion criteria, namely researching Gaming disorder.

\section{Screening and Eligibility}

In the search there were 27,513 article titles in science direct, 25,005 article titles in proquest and 2,187 titles in pubmed. The list of articles relevant to the research question was successfully identified. After screening through abstracts obtained 70 articles relevant to gaming disorder and adolescent emotional intelligence. The selection is continued by selecting journal articles that are relevant to the researcher's objectives, are eligible and have similar study design. In this final stage, there were 23 journal articles that met the criteria of the researchers' questions.

\section{Document Selection and Selection}

The selection of articles that have been included is a selection process based on existing inclusion and exclusion criteria. Inclusion and exclusion criteria have been determined by the author. Inclusion criteria include: there is an explanation of gaming behavior in adolescents, the impact that occurs from gaming disorder and adolescent emotional intelligence, the realm or scope of the article is community mental nursing, the assessment is getting stronger if the impact of gaming disorder can affect aspects of adolescent emotional intelligence, and articles use only English. In the exclusion criteria, namely the article's focus on the impact of addiction to gaming/gaming disorder, the method of the article is a systematic review and related article, cannot be read and cannot be edited. At this stage, the article needs to be read as a whole or full text. The results at the adjustment stage based on inclusion and exclusion criteria were 9 articles in the science direct database, 6 articles in the proquest database and 8 articles in the pubmed database. 

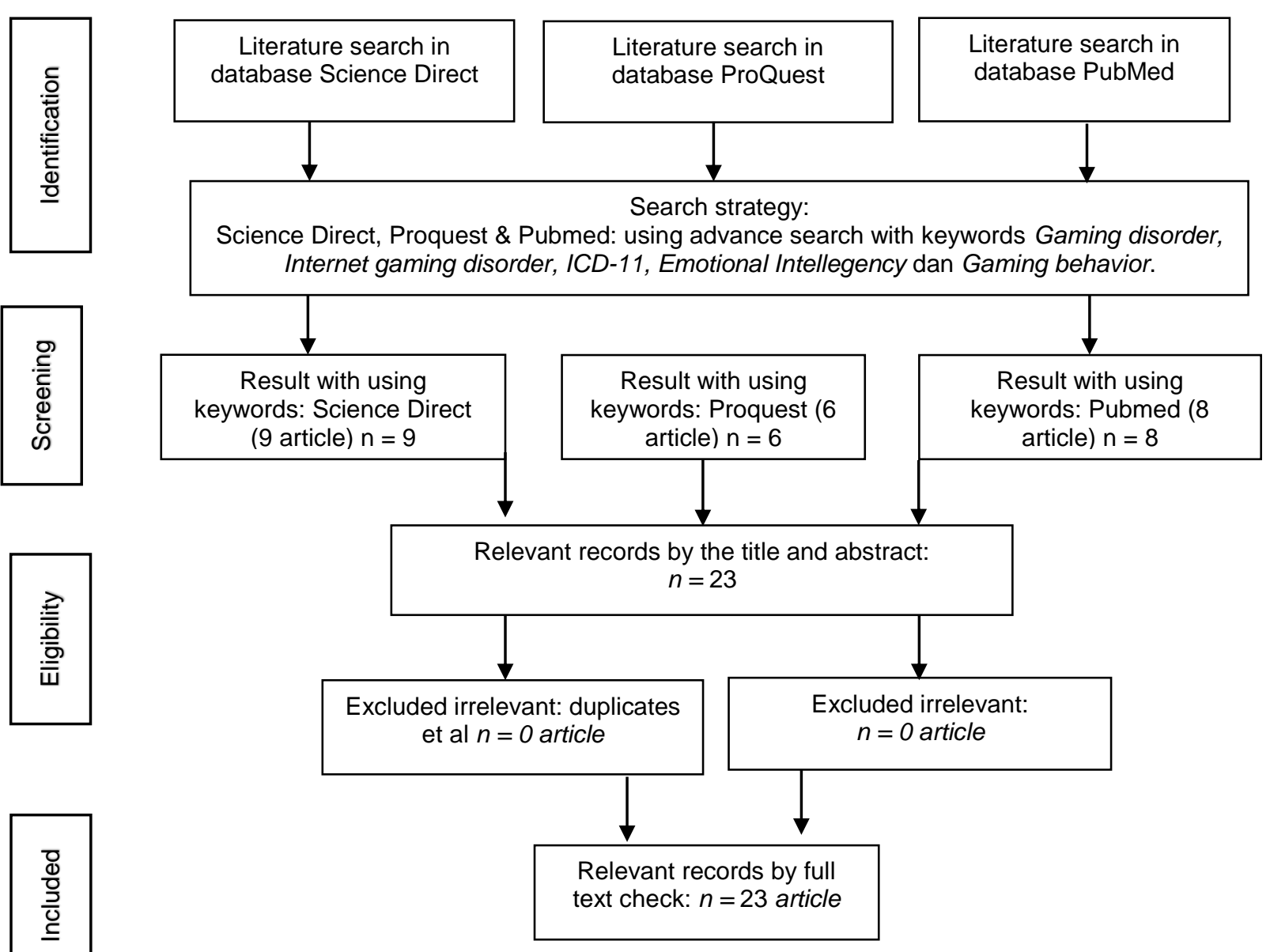

\section{Figure 1. Searching for Literature and the Process of Election Articles Modification of Prisma Flow Diagram}

\section{Aprasial}

The articles that have been obtained are then analyzed using the journal critic Joanna Brigg Institution (JBI) check list. Based on the appraisal carried out, a summary of the results of the study is attached to table 1 and synthesized into a systematic review. 
Table 1. Summary of Research Results on the Effects of Gaming Disorder

\begin{tabular}{|c|c|c|c|c|c|c|c|c|c|c|}
\hline No & $\begin{array}{c}\text { Authors } \\
\text { (Year) }\end{array}$ & Tittle & $\begin{array}{c}\text { Level } \\
\text { (JBI) }\end{array}$ & Aim & Method & Sample & Intervention & $\begin{array}{c}\text { Data } \\
\text { Analysis }\end{array}$ & Result & $\underline{\text { Aspect }}$ \\
\hline 1 & $\begin{array}{l}\text { Chia-Yi } \\
\text { Wu1, Ming- } \\
\text { Been Lee, } \\
\text { Shih-Cheng } \\
\text { Liao, Li- } \\
\text { Ren Chang } \\
\text { (2015) } \\
\text { U.S }\end{array}$ & $\begin{array}{l}\text { Risk Factors Of } \\
\text { Internet Addiction } \\
\text { Among Internet } \\
\text { Users: An Online } \\
\text { Questionnaire } \\
\text { Survey }\end{array}$ & JBI 4.a & $\begin{array}{l}\text { Knowing the } \\
\text { prevalence of } \\
\text { internet } \\
\text { addiction and } \\
\text { the } \\
\text { psychosocial } \\
\text { and } \\
\text { psychopatholo } \\
\text { gical } \\
\text { determinants } \\
\text { associated } \\
\text { with internet } \\
\text { users across all } \\
\text { age groups } \\
\end{array}$ & $\begin{array}{l}\text { Cross- } \\
\text { Sectional } \\
\text { Study }\end{array}$ & $\begin{array}{l}\text { A total of } \\
1100 \\
\text { respondents } \\
\text { consisted of } \\
156 \text { men, } 944 \\
\text { women }\end{array}$ & $\begin{array}{l}\text { The instrument } \\
\text { was given to } \\
\text { respondents with } \\
\text { a grid } \\
\text { CIAS-R), (BSRS- } \\
\text { 5), (MPI), and } \\
\text { questions about } \\
\text { suicide }\end{array}$ & $\begin{array}{l}\text { T-Test Or F- } \\
\text { Test }\end{array}$ & $\begin{array}{l}\text { Revealed that the results } \\
\text { significantly influence } \\
\text { neuroticism, life disorders } \\
\text { and time. (psychiatric } \\
\text { morbidity } 65 \% \text {, suicidal } \\
\text { ideation } 47 \% \text {, lifetime } \\
\text { suicide attempts } 23.1 \% \text { and } \\
\text { suicide attempts } 5.1 \% \text { a } \\
\text { year }\end{array}$ & \\
\hline 2 & $\begin{array}{l}\text { Qiao Liang, } \\
\text { Chengfu } \\
\text { Yu, } \\
\text { Quanfeng } \\
\text { Chen, } \\
\text { Xiaodong } \\
\text { Xie, Han } \\
\text { Wu, Jintao } \\
\text { Xing, } \\
\text { Shihua } \\
\text { Huang And } \\
\text { Kai Dou } \\
\text { (2019) } \\
\text { China }\end{array}$ & 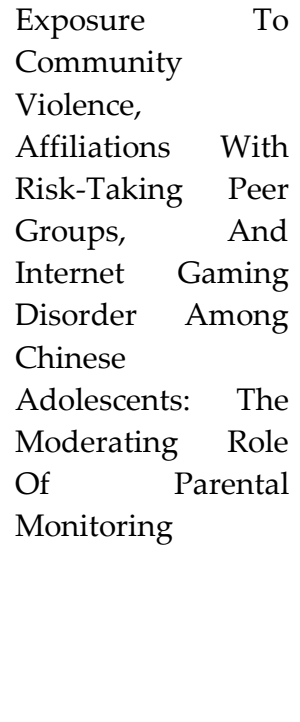 & JBI 2.a & $\begin{array}{l}\text { Test whether } \\
\text { parental } \\
\text { monitoring } \\
\text { moderates the } \\
\text { relationship } \\
\text { between ECV } \\
\text { and IGD } \\
\text { among } \\
\text { adolescents, } \\
\text { and whether } \\
\text { this } \\
\text { moderation } \\
\text { effect } \\
\text { mediated by } \\
\text { affiliations } \\
\text { with peers } \\
\text { who take risks. }\end{array}$ & $\begin{array}{l}\text { Stratified } \\
\text { And } \\
\text { Random } \\
\text { Cluster } \\
\text { Sampling } \\
\text { Techniques }\end{array}$ & $\begin{array}{l}\text { A sample } \\
\text { of } 2,423 \\
\text { Chinese } \\
\text { high school } \\
\text { students }\end{array}$ & $\begin{array}{l}\text { Data was } \\
\text { collected using } \\
\text { ECV } \\
\text { Questionnaire, } \\
\text { Parent Monitoring } \\
\text { Questionnaire, } \\
\text { Affiliation with } \\
\text { Risk Taking Peer } \\
\text { Group } \\
\text { Questionnaire, } \\
\text { IGD } \\
\text { Questionnaire, } \\
\text { and Impulsive } \\
\text { Scale. }\end{array}$ & $\begin{array}{l}\text { Compute } \\
\text { Correlations } \\
\text { And } \\
\text { Descriptive } \\
\text { Statistics }\end{array}$ & $\begin{array}{l}\text { These results indicate } \\
\text { adolescents are less able to } \\
\text { understand the } \\
\text { environment that is not } \\
\text { good so that makes teens } \\
\text { experience game } \\
\text { disruption. Close parental } \\
\text { monitoring can reduce the } \\
\text { impact of gaming disorder }\end{array}$ & $\begin{array}{c}\underline{\text { Self- }} \\
\text { awareness }\end{array}$ \\
\hline
\end{tabular}




\begin{tabular}{|c|c|c|c|c|c|c|c|c|c|c|}
\hline 3 & $\begin{array}{l}\text { Daniel L. } \\
\text { King, } \\
\text { Madeleine } \\
\text { C. E. Herd } \\
\text { And Paul } \\
\text { H. } \\
\text { Delfabbro } \\
\text { (2017) } \\
\text { Australia }\end{array}$ & $\begin{array}{l}\text { Tolerance } r \text { In } \\
\text { Internet Gaming } \\
\text { Disorder: A Need } \\
\text { For Increasing } \\
\text { Gaming Time Or } \\
\text { Something Else? }\end{array}$ & JBI 4.b & $\begin{array}{l}\text { The criteria in } \\
\text { DSM-5 } \\
\text { internet game } \\
\text { disruption } \\
\text { (IGD) refer to } \\
\text { the time spent } \\
\text { playing games }\end{array}$ & Random & $\begin{array}{l}630 \\
\text { Respondents } \\
\text { conducted } \\
\text { research }\end{array}$ & $\begin{array}{l}\text { The researcher } \\
\text { lets the } \\
\text { respondent adjust } \\
\text { all the rules that } \\
\text { the researcher has } \\
\text { set }\end{array}$ & $\begin{array}{l}\text { Analyzed } \\
\text { Using } \\
\text { Thematic } \\
\text { Analysis }\end{array}$ & $\begin{array}{l}\text { The results showed that } \\
\text { respondents with higher } \\
\text { game addiction resulted in } \\
\text { a large influence on mood. } \\
\text { Increasing desire, the need } \\
\text { to be involved in the game } \\
\text { due to game disruption. }\end{array}$ & \\
\hline 4 & $\begin{array}{l}\text { Süleyman } \\
\text { Çakiroğlu, } \\
\text { Nusret } \\
\text { Soylu } \\
\text { (2018) } \\
\text { Turki }\end{array}$ & $\begin{array}{l}\text { Adaptation Of } \\
\text { Internet Gaming } \\
\text { Disorder } \\
\text { Questionnaire To } \\
\text { Turkish: Reliability } \\
\text { And Validity Study }\end{array}$ & JBI 4.a & $\begin{array}{l}\text { This study } \\
\text { aims to } \\
\text { investigate the } \\
\text { validity and } \\
\text { reliability of } \\
\text { the Turkish } \\
\text { version of the } \\
\text { Internet } \\
\text { Gaming } \\
\text { Disorder } \\
\text { Questionnaire }\end{array}$ & $\begin{array}{l}\text { Validity } \\
\text { And } \\
\text { Reliability } \\
\text { Study }\end{array}$ & $\begin{array}{l}\text { 1161 } \\
\text { students } \\
\text { aged } \\
\text { between } 10 \\
\text { and } 18 \\
\text { from four } \\
\text { different } \\
\text { schools }\end{array}$ & $\begin{array}{l}\text { Using a } \\
\text { questionnaire } \\
\text { completed with } \\
\text { demographic data }\end{array}$ & $\begin{array}{l}\text { Test-Retest } \\
\text { Method And } \\
\text { Cronbach's } \\
\text { Alpha Internal } \\
\text { Consistency } \\
\text { Analysis }\end{array}$ & $\begin{array}{l}\text { The results obtained are } \\
\text { significant sub- } \\
\text { dimensional correlations of } \\
\text { the meaning of tolerance, } \\
\text { mood modification, } \\
\text { withdrawal, conflict and } \\
\text { recurrence. }\end{array}$ & \\
\hline 5 & $\begin{array}{l}\text { Sung Nyun } \\
\text { Kim And } \\
\text { Minah Kim } \\
(2018) \\
\text { Seoul }\end{array}$ & $\begin{array}{l}\text { Increased } \\
\text { Attentional Bias } \\
\text { Toward Visual } \\
\text { Cues In Internet } \\
\text { Gaming Disorder } \\
\text { And Obsessive- } \\
\text { Compulsive } \\
\text { Disorder: An } \\
\text { Event-Related } \\
\text { Potential Study } \\
\end{array}$ & JBI 4.b & $\begin{array}{l}\text { Analyzing the } \\
\text { effect of IGD } \\
\text { on nerve } \\
\text { function }\end{array}$ & $\begin{array}{l}\text { Qualitatif } \\
\text { Study. }\end{array}$ & $\begin{array}{l}\text { Twenty } \\
\text { patients } \\
\text { with IGD, } \\
20 \text { patients } \\
\text { with OCD, } \\
\text { and } 23 \\
\text { healthy } \\
\text { control } \\
\text { subjects } \\
(\mathrm{HC})\end{array}$ & $\begin{array}{l}\text { Presentation as a } \\
\text { researcher } \\
\text { provides a flow so } \\
\text { that respondents } \\
\text { are more able to } \\
\text { explore the correct } \\
\text { answer without } \\
\text { making or } \\
\text { deliberate. }\end{array}$ & $\begin{array}{l}\text { Independent } \\
\text { Sample T- } \\
\text { Tests }\end{array}$ & $\begin{array}{l}\text { The results of this study } \\
\text { indicate that game } \\
\text { disorders affect nerve } \\
\text { function. Internet gaming } \\
\text { disorder includes potential } \\
\text { addictions associated with } \\
\text { compulsive gaming } \\
\text { behavior. }\end{array}$ & \\
\hline 6 & $\begin{array}{l}\text { Tony } \\
\text { Durkee } \\
\text { And } \\
\text { Vladimir } \\
\text { Carli } \\
(2016)\end{array}$ & $\begin{array}{l}\text { Pathological } \\
\text { Internet Use And } \\
\text { Risk-Behaviors } \\
\text { Among European } \\
\text { Adolescents }\end{array}$ & JBI 4.a & $\begin{array}{l}\text { Investigate the } \\
\text { relationship } \\
\text { between } \\
\text { behavioral risk } \\
\text { and Piu } \\
\text { (pathological }\end{array}$ & $\begin{array}{l}\text { Cross- } \\
\text { Sectional } \\
\text { Study }\end{array}$ & $\begin{array}{l}89,281 \\
\text { Respondents }\end{array}$ & $\begin{array}{l}\text { Respondents were } \\
\text { given a } \\
\text { questionnaire and } \\
\text { were given a } \\
\text { vulnerability in } \\
\text { each answer to see }\end{array}$ & $\begin{array}{lr}\text { One-Way } & \\
\text { Analysis } & \text { Of } \\
\text { Variance } & \\
\text { (Anova) } & \text { With } \\
\text { Post } & \text { Hoc } \\
\text { Pairwise } & \\
\end{array}$ & $\begin{array}{l}\text { Shows that the risk } \\
\text { prevalence of problem } \\
\text { behavior is very significant } \\
\text { with the high intensity of } \\
\text { adolescents in internet use. } \\
\text { Influence is also found in }\end{array}$ & $\begin{array}{l}\underline{\text { Self- }} \\
\text { regulation }\end{array}$ \\
\hline
\end{tabular}




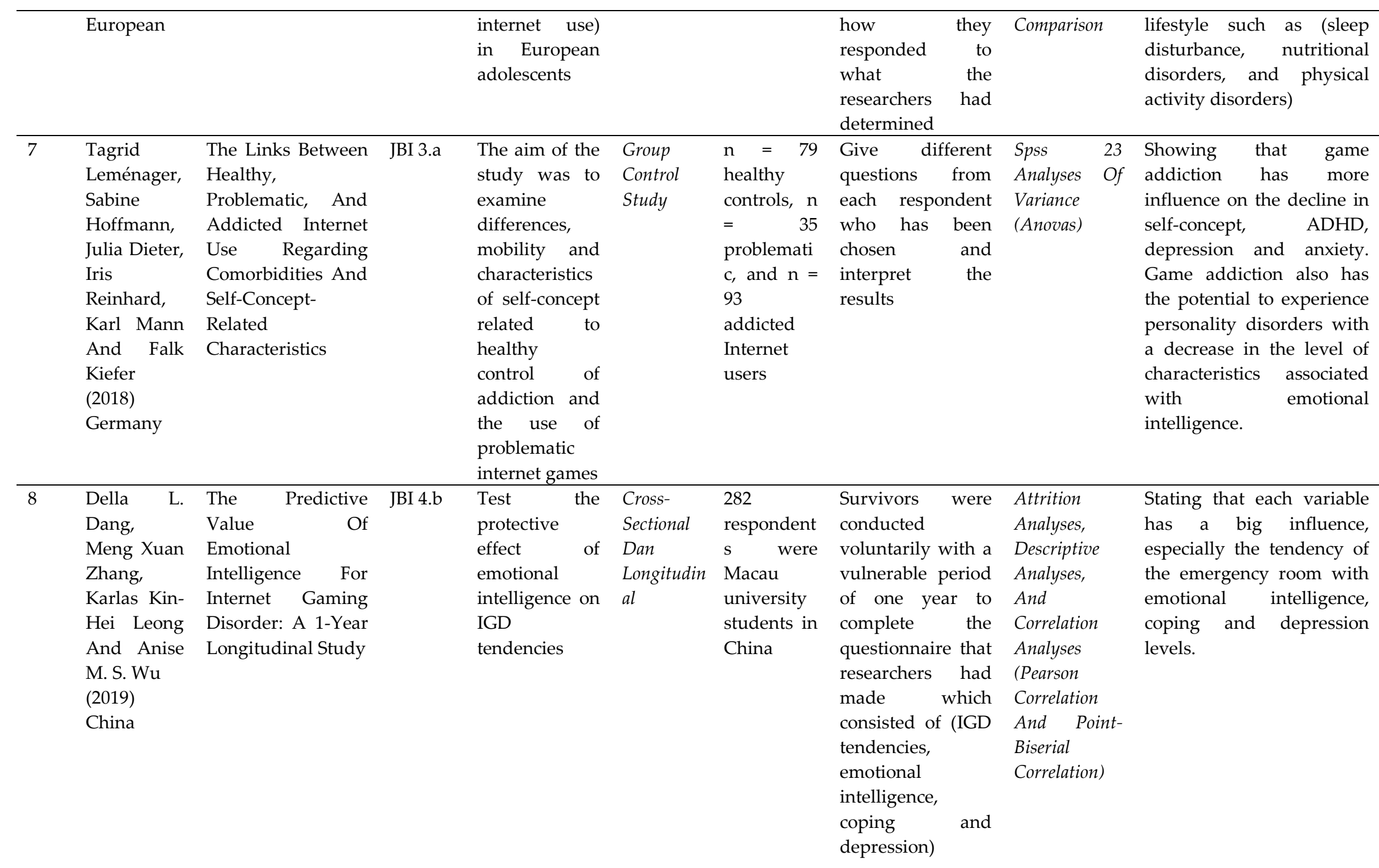




\begin{tabular}{|c|c|c|c|c|c|c|c|c|c|}
\hline 9 & $\begin{array}{l}\text { Guangheng } \\
\text { Dong, } \\
\text { Lingxiao } \\
\text { Wang, } \\
\text { Xiaoxia Du, } \\
\text { And Marc } \\
\text { N. Potenza } \\
\text { (2017) } \\
\text { Shanghai, } \\
\text { China }\end{array}$ & $\begin{array}{lr}\text { Gaming } & \text { Increases } \\
\text { Craving } & \text { To } \\
\text { Gaming-Related } \\
\text { Stimuli } \\
\text { Individuals } \quad \text { With } \\
\text { Internet Gaming } \\
\text { Disorder }\end{array}$ & JBI 4.a & $\begin{array}{l}\text { Analyzing the } \\
\text { relationship } \\
\text { between the } \\
\text { emergency } \\
\text { room with the } \\
\text { desire to play, } \\
\text { behavior and } \\
\text { neurology }\end{array}$ & $\begin{array}{l}\text { Correlatio } \\
n \text { Analyse }\end{array}$ & $\begin{array}{l}70 \\
\text { respondents }\end{array}$ & $\begin{array}{l}\text { Conduct } \\
\text { interviews with } \\
\text { each respondent }\end{array}$ & $\begin{array}{l}\text { Analyzed } \\
\text { Using Spm8 } \\
\text { And Neuroelf }\end{array}$ & $\begin{array}{l}\text { The result of IDG is the } \\
\text { loss of individual desire } \\
\text { control and changes in the } \\
\text { nervous system }\end{array}$ \\
\hline 10 & $\begin{array}{l}\text { Halley M. } \\
\text { Pontes 1,2,* } \\
\text { ' Bruno } \\
\text { Schivinski , } \\
\text { Magdalena } \\
\text { Brzozowsk } \\
\text { a-Wos' And } \\
\text { Vasileios } \\
\text { Stavropoul } \\
\text { os } \\
\text { (2019) } \\
\text { Australia }\end{array}$ & $\begin{array}{lr}\text { Laxer } & \text { Clinical } \\
\text { Criteria } & \text { For } \\
\text { Gaming } & \text { Disorder } \\
\text { May Hinder Future } \\
\text { Efforts } & \text { To Devise } \\
\text { An } & \text { Efficient } \\
\text { Diagnostic } & \\
\text { Approach: A Tree- } \\
\text { Based Model Study }\end{array}$ & JBI 4.a & $\begin{array}{l}\text { Analyze the } \\
\text { role played by } \\
\text { each } \\
\text { emergency } \\
\text { room criteria } \\
\text { in diagnosing } \\
\text { game } \\
\text { disorders }\end{array}$ & $\begin{array}{l}\text { Survey } \\
\text { Study }\end{array}$ & $\begin{array}{l}3377 \\
\text { participant } \\
\text { s (mean } \\
\text { age } \quad 20 \\
\text { years, } \\
\text { elementary } \\
\text { school = } 4.3 \\
\text { years) }\end{array}$ & $\begin{array}{l}\text { Data were } \\
\text { collected using a } \\
\text { questionnaire } \\
\text { with the nine-item } \\
\text { IGDS9-SF } \\
\text { provisions }\end{array}$ & $\begin{array}{ll}\text { MCAR } & \text { Test } \\
\text { And } & \text { Chi- } \\
\text { Square } & \end{array}$ & $\begin{array}{l}\text { Gaming disorder says } \\
\text { gamers lose control of } \\
\text { themselves. Without the } \\
\text { support to control or stop } \\
\text { it will continue }\end{array}$ \\
\hline 11 & $\begin{array}{l}\text { Hyunsuk } \\
\text { Jeong, } \\
\text { Hyeon Woo } \\
\text { Yim, } \\
\text { Seung-Yup } \\
\text { Lee, Hae } \\
\text { Kook Lee, } \\
\text { Marc N. } \\
\text { Potenza, } \\
\text { Jung-Hye } \\
\text { Kwon, } \\
\text { Hoon Jung } \\
\text { Koo, Yong- } \\
\end{array}$ & $\begin{array}{lr}\text { Discordance } & \\
\text { Between } & \text { Self- } \\
\text { Report } & \\
\text { And } & \text { Clinical } \\
\text { Diagnosis } & \text { Of } \\
\text { Internet } & \\
\text { Gaming } & \text { Disorder } \\
\text { In Adolescents }\end{array}$ & 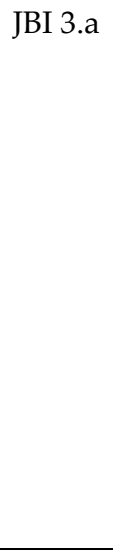 & $\begin{array}{l}\text { Estimating the } \\
\text { level of } \\
\text { overreporting } \\
\text { (false positive) } \\
\text { and } \\
\text { underreportin } \\
\mathrm{g} \text { (false } \\
\text { negative) }\end{array}$ & $\begin{array}{l}\text { Case-Sub- } \\
\text { Cohort }\end{array}$ & $\begin{array}{l}45 \text { with } \\
\text { IGD and } \\
228 \\
\text { without } \\
\text { IGD }\end{array}$ & $\begin{array}{l}\text { Distribute } \\
\text { questionnaires } \\
\text { that include } \\
\text { respondent } \\
\text { characteristic data } \\
\text { and Self-report } \\
\text { assessment }\end{array}$ & ANOVA Test & $\begin{array}{l}\text { Respondents with gaming } \\
\text { disorder cause high } \\
\text { anxiety, suicidal ideation, } \\
\text { aggression, poor self- } \\
\text { control, low self-esteem. As } \\
\text { a result of too much time is } \\
\text { used to play games }\end{array}$ \\
\hline
\end{tabular}




\begin{tabular}{|c|c|c|c|c|c|c|c|c|c|c|}
\hline & $\begin{array}{l}\text { Sil Kweon, } \\
\text { Soo-Young } \\
\text { Bhang \& } \\
\text { Jung-Seok } \\
\text { Choi } \\
(2018) \\
\text { Korea }\end{array}$ & & & & & & & & & \\
\hline 12 & $\begin{array}{l}\text { G.H. } \\
\text { Schoenmac } \\
\text { ker, A.P. } \\
\text { Groenman, } \\
\text { E. Sokolov, } \\
\text { J. } \\
\text { Oosterlaan, } \\
\text { N. } \\
\text { Rommelse, } \\
\text { H. Roeyers, } \\
\text { R.D. Oades' } \\
\text { S.V. } \\
\text { Faraone B. } \\
\text { Franke T. } \\
\text { Heskes, A. } \\
\text { Arias } \\
\text { Vasquez, T. } \\
\text { Claasse, } \\
\text { J.K. } \\
\text { Buitelaar } \\
\text { (2018) } \\
\text { Netherland } \\
\text { s }\end{array}$ & $\begin{array}{l}\text { Role Of Conduct } \\
\text { Problems In The } \\
\text { Relation Between } \\
\text { Attention-Deficit } \\
\text { Hyperactivity } \\
\text { Disorder, } \\
\text { Substance Use, } \\
\text { And Gaming }\end{array}$ & JBI 4.d & $\begin{array}{l}\text { Establish a } \\
\text { causal model } \\
\text { of the } \\
\text { relationship } \\
\text { between } \\
\text { ADHD and } \\
\text { comorbid } \\
\text { behavior } \\
\text { problems, and } \\
\text { alcohol, } \\
\text { nicotine, and } \\
\text { other } \\
\text { substance use }\end{array}$ & $\begin{array}{l}\text { Case- } \\
\text { Only } \\
\text { Study }\end{array}$ & $\begin{array}{l}362 \\
\text { individuals } \\
\text { diagnosed } \\
\text { with } \\
\text { ADHD at } \\
\text { the age of } \\
12-24 \text { years }\end{array}$ & $\begin{array}{l}\text { Questionnaire } \\
\text { with each aspect } \\
\text { in accordance } \\
\text { with the } \\
\text { objectives of the } \\
\text { researcher }\end{array}$ & $\begin{array}{l}\text { Bayesian } \\
\text { Constraint- } \\
\text { Based Causal } \\
\text { Discovery } \\
(\text { Bccd })\end{array}$ & $\begin{array}{l}\text { That dependency on } \\
\text { playing games has a causal } \\
\text { path } \\
\text { Related to ADHD }\end{array}$ & \\
\hline 13 & $\begin{array}{l}\text { Muhannad } \\
\text { Quwaider, } \\
\text { Abdullah } \\
\text { Alabed } \\
\text { And Rehab } \\
\text { Duwairi }\end{array}$ & $\begin{array}{l}\text { The Impact Of } \\
\text { Video Games On } \\
\text { The Players } \\
\text { Behaviors: } \\
\text { Survey }\end{array}$ & JBI 4.a & $\begin{array}{l}\text { Know the } \\
\text { relationship } \\
\text { between game } \\
\text { addiction and } \\
\text { game player } \\
\text { behavior }\end{array}$ & $\begin{array}{l}\text { Qualitatif } \\
\text { Study. }\end{array}$ & $\begin{array}{l}205 \\
\text { students }\end{array}$ & $\begin{array}{l}\text { Given } \\
\text { questionnaires } \\
\text { and conduct } \\
\text { interviews }\end{array}$ & A Survey & $\begin{array}{l}\text { Game addiction makes } \\
\text { student motivation } \\
\text { becomes low, students do } \\
\text { not have the desire to be } \\
\text { smarter or seek } \\
\text { knowledge. }\end{array}$ & 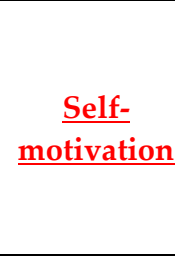 \\
\hline
\end{tabular}




\begin{tabular}{|c|c|c|c|c|c|c|c|c|c|c|}
\hline & $\begin{array}{l}\text { (2019) } \\
\text { Jordan }\end{array}$ & & & & & & & & & \\
\hline 14 & $\begin{array}{l}\text { Vasileios } \\
\text { Stavropoul } \\
\text { os, Emma } \\
\text { Ela } \\
\text { Anderson, } \\
\text { Charlotte } \\
\text { Beard, } \\
\text { Mohamme } \\
\text { d Qasim } \\
\text { Latifi, } \\
\text { Daria Kuss, } \\
\text { Mark } \\
\text { Griffiths } \\
\text { (2019) } \\
\text { Australia }\end{array}$ & $\begin{array}{l}\text { A Preliminary } \\
\text { Cross-Cultural } \\
\text { Study Of } \\
\text { Hikikomori And } \\
\text { Internet Gaming } \\
\text { Disorder: The } \\
\text { Moderating Effects } \\
\text { Of Game-Playing } \\
\text { Time And Living } \\
\text { With Parents }\end{array}$ & JBI 4.a & $\begin{array}{l}\text { Analyzing the } \\
\text { relationship } \\
\text { between } \\
\text { Hikikomori } \\
\text { and the ER, } \\
\text { and the } \\
\text { potential } \\
\text { moderate } \\
\text { effect of } \\
\text { playing games } \\
\text { and living } \\
\text { with parents. }\end{array}$ & $\begin{array}{l}\text { Cross- } \\
\text { Sectional } \\
\text { Study }\end{array}$ & $\begin{array}{l}153 \text { young } \\
\text { adult } \\
\text { players are } \\
\text { from } \\
\text { Australia } \\
\text { and } 457 \\
\text { adults are } \\
\text { from the } \\
\text { U.S. North } \\
\text { America }\end{array}$ & $\begin{array}{l}\text { Questionnaires } \\
\text { were given based } \\
\text { on the Nine Scale } \\
\text { Internet Gaming } \\
\text { Disorder-Short } \\
\text { Form (IGDS-SF9), } \\
\text { and the } \\
\text { Hikikomori Social } \\
\text { Withdrawal Scale }\end{array}$ & $\begin{array}{l}\text { Linear } \\
\text { Regression } \\
\text { Analyses }\end{array}$ & $\begin{array}{l}\text { The results show that, the } \\
\text { occurrence of withdrawal } \\
\text { or (Hikikomori) } \\
\text { experienced by young } \\
\text { adults is closely related to } \\
\text { game addiction. }\end{array}$ & \\
\hline 15 & $\begin{array}{l}\text { Socayna } \\
\text { Moudiab, } \\
\text { Marcantoni } \\
\text { o M. Spada } \\
\text { (2019) } \\
\text { London, Uk }\end{array}$ & $\begin{array}{l}\text { The Relative } \\
\text { Contribution Of } \\
\text { Motives And } \\
\text { Maladaptive } \\
\text { Cognitions To } \\
\text { Levels Of Internet } \\
\text { Gaming Disorder }\end{array}$ & 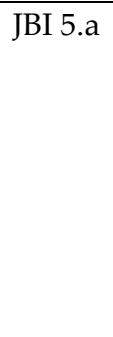 & $\begin{array}{l}\text { Identifying } \\
\text { maladaptive } \\
\text { motivation } \\
\text { and cognitive }\end{array}$ & $\begin{array}{l}\text { Correlation } \\
\text { Analyse }\end{array}$ & $\begin{array}{l}79 \\
\text { participant } \\
\mathrm{s} \quad \text { in } \\
\text { London, } \\
\text { UK }\end{array}$ & $\begin{array}{l}\text { Using a short } \\
\text { questionnaire } \\
\text { form }\end{array}$ & $\begin{array}{l}\text { A Pearson } \\
\text { Product } \\
\text { Moment } \\
\text { Correlation } \\
\text { Linear } \\
\text { Regression } \\
\text { Analysis }\end{array}$ & $\begin{array}{l}\text { Each item has a close } \\
\text { relationship between game } \\
\text { addiction that results in } \\
\text { decreased mitivation and } \\
\text { maladaptive cognitive }\end{array}$ & \\
\hline 16 & $\begin{array}{l}\text { Tobias } \\
\text { Greitemeye } \\
\text { r } \\
(2018) \\
\text { Austria }\end{array}$ & $\begin{array}{lr}\text { The } & \text { Spreading } \\
\text { Impact } & \text { Of } \\
\text { Playing } \\
\text { Violent } & \text { Video } \\
\text { Games } & \text { On } \\
\text { Aggression } & \end{array}$ & JBI 5.a & $\begin{array}{l}\text { Analyze the } \\
\text { existence of } \\
\text { violent } \\
\text { behavior and } \\
\text { aggression } \\
\text { resulting from } \\
\text { game } \\
\text { addiction }\end{array}$ & $\begin{array}{l}\text { Descriptive } \\
\text { Analysis }\end{array}$ & $\begin{array}{l}499 \text { male, } \\
499 \text { female }\end{array}$ & $\begin{array}{l}\text { Given } \\
\text { demographic } \\
\text { questionnaire, } \\
\text { aggressive } \\
\text { behavior. Each } \\
\text { participant } \\
\text { receives } 10 \text { items }\end{array}$ & A Sobel Test & $\begin{array}{l}\text { Game addiction is proven } \\
\text { to increase violent } \\
\text { behavior and acts of } \\
\text { aggression. }\end{array}$ & Self Empathy \\
\hline 17 & $\begin{array}{l}\text { Joe } \quad \text { L } \\
\text { Billieux, }\end{array}$ & $\begin{array}{lr}\text { Cognitive } & \text { Factors } \\
\text { Associated } & \text { With }\end{array}$ & JBI 3.d & $\begin{array}{l}\text { Analyze the } \\
\text { causal factors }\end{array}$ & $\begin{array}{l}\text { Case- } \\
\text { Control }\end{array}$ & $\begin{array}{l}56 \\
\text { individuals }\end{array}$ & $\begin{array}{l}\text { Using a } \\
\text { documentation }\end{array}$ & $\begin{array}{l}\text { Descriptive } \\
\text { Analysis }\end{array}$ & $\begin{array}{l}\text { Factors resulting from } \\
\text { game addiction include: }\end{array}$ & \\
\hline
\end{tabular}




\begin{tabular}{|c|c|c|c|c|c|c|c|c|}
\hline & $\begin{array}{l}\text { Marc N. } \\
\text { Potenza, } \\
\text { Pierre } \\
\text { Maurage, } \\
\text { Damien } \\
\text { Brevers, } \\
\text { Matthias } \\
\text { Brand' And } \\
\text { Daniel L. } \\
\text { King (2019) } \\
\text { Luxemburg }\end{array}$ & Gaming Disorder & $\begin{array}{l}\text { of game } \\
\text { addiction }\end{array}$ & Designs & $\begin{array}{l}\text { and } \quad 61 \\
\text { clinic } \\
\text { respondent } \\
\mathrm{s}\end{array}$ & $\begin{array}{l}\text { system to observe } \\
\text { cognitive, } \\
\text { affective and } \\
\text { psychomotor }\end{array}$ & & $\begin{array}{l}\text { loss of feelings or } \\
\text { sensitivity to the } \\
\text { environment and social, } \\
\text { the emergence of high } \\
\text { ignorance, strengthening } \\
\text { sensitivity, and executive } \\
\text { function }\end{array}$ \\
\hline 18 & $\begin{array}{l}\text { Cuneyt } \\
\text { Evren, } \\
\text { Bilge } \\
\text { Evren, } \\
\text { Ercan } \\
\text { Dalbudak , } \\
\text { Merve } \\
\text { Topcu , } \\
\text { Nilay Kutlu } \\
\text { (2019) } \\
\text { Turkie }\end{array}$ & $\begin{array}{l}\text { Relationship Of JBI 5.a } \\
\text { Internet Gaming } \\
\text { Disorder Severity } \\
\text { With Symptoms Of } \\
\text { Anxiety, } \\
\text { Depression, } \\
\text { Alexithymia, And } \\
\text { Aggression Among } \\
\text { University } \\
\text { Students }\end{array}$ & $\begin{array}{l}\text { The purpose } \\
\text { of this study } \\
\text { was to } \\
\text { evaluate the } \\
\text { relationship of } \\
\text { the severity of } \\
\text { internet } \\
\text { gaming } \\
\text { disorders with } \\
\text { alexithymia } \\
\text { And } \\
\text { aggression } \\
\text { among } \\
\text { students, } \\
\text { while } \\
\text { controlling the } \\
\text { effects of } \\
\text { anxiety and } \\
\text { depressive } \\
\text { symptoms. }\end{array}$ & $\begin{array}{l}\text { Online } \\
\text { Survey }\end{array}$ & $\begin{array}{l}987 \\
\text { university } \\
\text { volunteer } \\
\text { students in } \\
\text { Ankara }\end{array}$ & $\begin{array}{l}\text { Participants were } \\
\text { evaluated by } \\
\text { managing the } \\
\text { Toronto } \\
\text { Alexithymia Scale } \\
\text { (TAS-20), the } \\
\text { Buss-Perry } \\
\text { Aggressive } \\
\text { Questionnaire } \\
\text { (BPAQ), the } \\
\text { Gaming-Short- } \\
\text { Form Internet } \\
\text { Disruption Scale } \\
\text { (IGDS9-SF), and } \\
\text { the depression } \\
\text { and anxiety } \\
\text { subscale of the 90- } \\
\text { Revised Symptom } \\
\text { Item Checklist } \\
\text { (SCL-90-R) }\end{array}$ & $\begin{array}{l}\text { Linear } \\
\text { Regression } \\
\text { Analysis }\end{array}$ & $\begin{array}{l}\text { This finding shows that } \\
\text { among students } \\
\text { The effect of more gaming } \\
\text { disorders is alexithymia } \\
\text { "difficulty identifying } \\
\text { feelings" }\end{array}$ \\
\hline 19 & $\begin{array}{l}\text { Daniel L. } \\
\text { King, Cam } \\
\text { Adair, John } \\
\text { B. } \\
\end{array}$ & $\begin{array}{lrr}\text { Clinical } & \text { Predictors } & \text { JBI 2.a } \\
\text { Of } \quad \text { Gaming } & \\
\text { Abstinence In } & \\
\text { Help-Seeking } & \end{array}$ & $\begin{array}{l}\text { Analyzing the } \\
\text { effectiveness } \\
\text { of } \\
\text { interventions }\end{array}$ & $\begin{array}{l}\text { Follow Up } \\
\text { Survey }\end{array}$ & $\begin{array}{l}186 \text { adult } \\
\text { gamers }\end{array}$ & $\begin{array}{l}\text { Providing } \\
\text { questionnaires } \\
\text { related to DSM-5 } \\
\text { Internet gaming }\end{array}$ & $\begin{array}{l}\text { Mann- } \\
\text { Whitney U } \\
\text { Tests And } \\
\text { Chi-Square } \\
\end{array}$ & $\begin{array}{l}\text { Respondents experience } \\
\text { mood disorders and poor } \\
\text { quality of life }\end{array}$ \\
\hline
\end{tabular}




\begin{tabular}{|c|c|c|c|c|c|c|c|c|c|c|}
\hline & $\begin{array}{l}\text { Saunders, } \\
\text { Paul H. } \\
\text { Delfabbro } \\
(2018) \\
\text { Canada }\end{array}$ & $\begin{array}{l}\text { Adult Problematic } \\
\text { Gamers }\end{array}$ & & $\begin{array}{l}\text { for } \\
\text { problematic } \\
\text { games due to } \\
\text { the IGD }\end{array}$ & & & \begin{tabular}{lr} 
disorder & $(\mathrm{IGD})$, \\
Depression & \\
Anxiety & Stress \\
Scales-21, Internet \\
Cognition & Scale, \\
Gaming & Craving \\
Scale, & and \\
Gaming & Quality \\
\multicolumn{2}{c}{ of Life Scale } \\
\end{tabular} & Tests & & \\
\hline 20 & $\begin{array}{l}\text { Wavne } \\
\text { Rikkers, } \\
\text { David } \\
\text { Lawrence, } \\
\text { Jennifer } \\
\text { Hafekost } \\
\text { And } \\
\text { Stephen R. } \\
\text { Zubrick } \\
\text { (2016) } \\
\text { Australia }\end{array}$ & $\begin{array}{l}\text { Internet Use And } \\
\text { Electronic Gaming } \\
\text { By Children And } \\
\text { Adolescents With } \\
\text { Emotional And } \\
\text { Behavioural } \\
\text { Problems In } \\
\text { Australia - Results } \\
\text { From The Second } \\
\text { Child And } \\
\text { Adolescent Survey } \\
\text { Of Mental Health } \\
\text { And Wellbeing } \\
\end{array}$ & JBI 4.a & $\begin{array}{l}\text { Explain the } \\
\text { current } \\
\text { internet usage } \\
\text { and behavior } \\
\text { of electronic } \\
\text { games that } \\
\text { have an } \\
\text { impact on } \\
\text { mental } \\
\text { disorders }\end{array}$ & $\begin{array}{l}\text { Random } \\
\text { Sampling }\end{array}$ & $\begin{array}{l}\text { A total of } \\
2967 \\
\text { respondent } \\
\mathrm{s} \text { aged } 11- \\
17 \text { years }\end{array}$ & $\begin{array}{l}\text { The survey was } \\
\text { conducted based } \\
\text { on how much } \\
\text { time the } \\
\text { respondent spent, } \\
\text { measurements } \\
\text { were made every } \\
2 \text { hours during the } \\
\text { survey process }\end{array}$ & $\begin{array}{l}\text { Regression } \\
\text { Logistic } \\
\text { Multivariat }\end{array}$ & $\begin{array}{lc}\text { Teenagers } & \text { suffer from } \\
\text { emotional problems or } \\
\text { high } & \text { psychological } \\
\text { pressure. resulting in poor } \\
\text { social relations. }\end{array}$ & 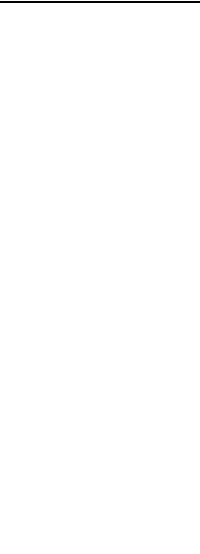 \\
\hline 21 & $\begin{array}{l}\text { Netta } \\
\text { Weinstein, } \\
\text { Andrew K. } \\
\text { Przybylski } \\
\text { And Kou } \\
\text { Murayama } \\
\text { (2017) } \\
\text { U.K }\end{array}$ & $\begin{array}{l}\text { A Prospective } \\
\text { Study Of The } \\
\text { Motivational And } \\
\text { Health Dynamics } \\
\text { Of Internet Gaming } \\
\text { Disorder }\end{array}$ & JBI 4.b & $\begin{array}{l}\text { Investigate the } \\
\text { etiology and } \\
\text { personal } \\
\text { results of } \\
\text { internet } \\
\text { gaming } \\
\text { disorder }\end{array}$ & $\begin{array}{l}\text { Sensitivity } \\
\text { Analysis } \\
\text { Explora-tory } \\
\text { Analyses }\end{array}$ & $\begin{array}{l}\text { Responden } \\
\text { ts were } \\
5777 \text { aged } \\
18 \text { years }\end{array}$ & $\begin{array}{l}\text { Respondents were } \\
\text { given questions } \\
\text { and were also } \\
\text { asked to do what } \\
\text { the researchers } \\
\text { had planned, } \\
\text { which aims to see } \\
\text { the criteria and } \\
\text { the angle of the } \\
\text { response field to } \\
\text { the methods and } \\
\text { framework that } \\
\text { the researchers } \\
\text { had determined }\end{array}$ & $\begin{array}{l}\text { Sem } \\
\text { Negative } \\
\text { Binomial } \\
\text { Regression } \\
\text { Analysis }\end{array}$ & $\begin{array}{l}\text { Consistent results indicate } \\
\text { that the negative impact of } \\
\text { internet gaming disorder } \\
\text { occurs on social activities } \\
\text { and basic needs. }\end{array}$ & $\underline{\text { Social Skills }}$ \\
\hline
\end{tabular}




\begin{tabular}{|c|c|c|c|c|c|c|c|c|c|}
\hline 22 & $\begin{array}{l}\text { Chih-Hung } \\
\text { Ko1,2,3, } \\
\text { Huang-Chi } \\
\text { Lin3,4, Pai- } \\
\text { Cheng } \\
\text { Lin4,5 And } \\
\text { Ju-Yu Yen } \\
\text { (2019) } \\
\text { Australian } \\
\text { And } \\
\text { New } \\
\text { Zealand }\end{array}$ & $\begin{array}{l}\text { Validity, } \\
\text { Functional } \\
\text { Impairment And } \\
\text { Complications } \\
\text { Related To Internet } \\
\text { Gaming Disorder } \\
\text { In The DSM-5 And } \\
\text { Gaming Disorder } \\
\text { In The ICD-11 }\end{array}$ & JBI 3.d & $\begin{array}{l}\text { Evaluating } \\
\text { functional } \\
\text { disorders, } \\
\text { unhealthy } \\
\text { behaviors and } \\
\text { complications } \\
\text { among adults } \\
\text { with internet } \\
\text { game } \\
\text { disorders and } \\
\text { game } \\
\text { disorders. }\end{array}$ & $\begin{array}{l}\text { Case } \\
\text { Control }\end{array}$ & $\begin{array}{l}69 \text { regular } \\
\text { gamers } \\
\text { and } 69 \\
\text { controls }\end{array}$ & $\begin{array}{l}\text { Conducting } \\
\text { interviews, } \\
\text { Interviewing } \\
\text { conducted by a } \\
\text { psychiatrist } \\
\text { according to the } \\
\text { DSM-5 Internet } \\
\text { gaming disorder } \\
\text { criteria }\end{array}$ & $\begin{array}{l}\text { One-Way } \\
\text { Analysis }\end{array}$ & $\begin{array}{l}\text { The results obtained } \\
\text { respondents have impaired } \\
\text { academic function, work or } \\
\text { social. }\end{array}$ \\
\hline 23 & $\begin{array}{l}\text { Hee Joung } \\
\text { Seok, } \\
\text { Jeoung Min } \\
\text { Lee, Chi- } \\
\text { Yong Park, } \\
\text { Ji Young } \\
\text { Park } \\
(2018) \\
\text { Korea }\end{array}$ & $\begin{array}{l}\text { Understanding } \\
\text { Internet Gaming } \\
\text { Addiction Among } \\
\text { South Korean } \\
\text { Adolescents } \\
\text { Through } \\
\text { Photovoice }\end{array}$ & 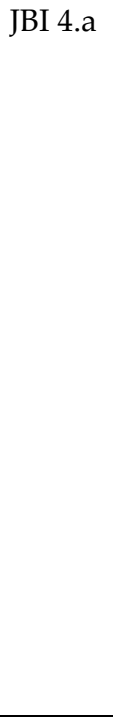 & $\begin{array}{l}\text { Explore the } \\
\text { motivation of } \\
\text { teenagers for } \\
\text { internet } \\
\text { games, how } \\
\text { their lives are } \\
\text { affected, how } \\
\text { they view } \\
\text { internet } \\
\text { games, what } \\
\text { they have } \\
\text { gained and } \\
\text { lost, and how } \\
\text { they } \\
\text { understand } \\
\text { internet game } \\
\text { addiction. }\end{array}$ & $\begin{array}{l}\text { Qualitative } \\
\text { Study. }\end{array}$ & $\begin{array}{l}10 \\
\text { teenagers } \\
\text { are at risk } \\
\text { of IGD }\end{array}$ & $\begin{array}{l}\text { Interviews, focus } \\
\text { groups, and photo } \\
\text { invoices } \\
\text { All respondents }\end{array}$ & $\begin{array}{l}\text { Utilized } \\
\text { Photovoice } \\
\text { Study }\end{array}$ & $\begin{array}{l}\text { These results indicate that } \\
\text { regard internet games as a } \\
\text { way for entertainment, } \\
\text { stress relief, and peer bond. }\end{array}$ \\
\hline
\end{tabular}




\section{RESULTS AND DISCUSSION}

Based on the questions made by researchers namely how the impact of gaming disorder on adolescent emotional intelligence, the results show mixed data. The different results are adapted to the system, and the conditions of the country or place of each study. The impact of gaming disorder is indeed very significant on each aspect of adolescent emotional intelligence.

The source obtained clearly seen that the effects of gaming disorder interfere with adolescent emotional intelligence. The most impact on emotional intelligence is on aspects of self-regulation. Gaming disorder increases lifestyle disorders, suicidal ideas, compulsive behaviors, loss of self-control and disruption of physical activity (Dang, Zhang, Leong, \& Wu, 2019; Dong, Wang, Du, \& Potenza, 2017; Jeong et al., 2018; Pontes, Schivinski, Brzozowska-woś, \& Stavropoulos, 2019; Schoenmacker et al., 2018; Seok, Lee, Park, \& Park, 2018; Stavropoulos et al., 2019). As a result of gaming disorder makes adolescents lose self-regulation which should be developed in adolescence to be more able to control themselves well.

The impact on aspects of self-awareness resulting from gaming disorder is making it difficult for adolescents to understand environmental conditions, the lack of excessive desire and affect self-understanding (Kim et al., 2018; King, Herd, \& Delfabbro, 2017; Liang et al., 2019; Çakiroğlu, 2018; Wu, Lee, Liao, \& Chang, 2015). The aspect of self-awareness on emotional intelligence also has a big influence on adolescents, because if it has been affected by gaming disorder it will make teenagers more difficult to understand themselves. Aspects of self-motivation in adolescents who experience gaming disorder resulting in a decrease in the learning process, withdrawal and reduce the desire to seek knowledge (Moudiab \& Spada, 2019; Quwaider, Alabed, \& Duwairi, 2019; Stavropoulos et al., 2019).

The self-empathy aspects of teen gaming disorder do not have sensitivity to the environment, violent behavior and can suffer from alexithymia (Evren, Dalbudak, Topcu, \& Kutlu, 2019; Greitemeyer, 2018; King et al., 2018; Potenza, Maurage, Brevers, Brand, \& King, 2019). The impact of gaming disorder on aspects of social skills on emotional intelligence results in poor social relations, decreases interest in social activities, loss of entertainment in the environment and disruption of academic and social functions (Ko, Lin, Lin, \& Yen, 2019; Rikkers, Lawrence, Hafekost, \& Zubrick, 2016; Seok et al., 2018; Weinstein, Przybylski, \& Murayama, 2017).

This means that gaming disorder is indeed worthy of being used as a patent diagnosis for mental disorders. The impact caused by gaming disorder has a lot of empirical evidence which in the future also requires further treatment for sufferers of gaming disorder in the future.

\section{CONCLUSION}

The impact of gaming disorder on adolescent emotional intelligence for now really needs to be aware of, because not a few problems that afflict adolescents due to gaming. WHO regulation to include gaming disorder as ICD-11 is indeed very 
appropriate with the increasing number of problems due to gaming disorder. Gaming disorder is the main trigger that affects the emotional intelligence of adolescents today. The highest aspect due to gaming disorder is in the aspect of selfregulation, but aspects of self-awareness, self-empathy, self-motivation and social skills also have a significant impact. Emotional intelligence is very important to be mastered in life, if emotional intelligence has been disturbed due to gaming disorder then the five aspects of emotional intelligence will also be disturbed.

\section{REFERENCES}

1. American Psychiatric Association (2013). DSM 5. American Psychiatric Association.

2. Ayenigbara, I. (2018). Gaming Disorder and Effects of Gaming on Health: An Overview. Journal of Addiction Medicine and Therapeutic Science, 4, 001-003. https://doi.org/10.17352/2455-3484.000025

3. Dang, D. L., Zhang, M. X., Leong, K. K., \& Wu, A. M. S. (2019). The Predictive Value of Emotional Intelligence for Internet Gaming Disorder: A 1-Year Longitudinal Study. 12-15.

4. Dong, G., Wang, L., Du, X., \& Potenza, M. N. (2017). Gaming Increases Craving to Gaming-Related Stimuli in Individuals With Internet Gaming Disorder. Biological Psychiatry: Cognitive Neuroscience and Neuroimaging, 2(5), 404-412. https://doi.org/10.1016/j.bpsc.2017.01.002

5. Evren, C., Evren, B., Dalbudak, E., Topcu, M., \& Kutlu, N. (2019). Relationship of internet gaming disorder severity with symptoms of anxiety, depression, alexithymia, and aggression among university students. Dusunen Adam, 32(3), 227-235. https://doi.org/10.14744/DAJPNS.2019.00032

6. Greitemeyer, T. (2018). The spreading impact of playing violent video games on aggression. Computers in Human Behavior, 80, 216-219. https://doi.org/10.1016/j.chb.2017.11.022

7. Jeong, H., Yim, H. W., Lee, S. Y., Lee, H. K., Potenza, M. N., Kwon, J. H., ... Choi, J. S. (2018). Discordance between self-report and clinical diagnosis of Internet gaming disorder in adolescents. Scientific Reports, 8(1), 1-9. https://doi.org/10.1038/s41598-018-28478-8

8. Kim, S. N., Kim, M., Lee, T. H., Lee, J. Y., Park, S., Park, M., ... Choi, J. S. (2018). Increased attentional bias toward visual cues in internet gaming disorder and obsessive-compulsive disorder: An event-related potential study. Frontiers in Psychiatry, 9(JUL), 1-9. https://doi.org/10.3389/fpsyt.2018.00315

9. King, D. L., Adair, C., Saunders, J. B., Delfabbro, P. H., King, D. L., Adair, C., ... Paul, H. (2018). Clinical predictors of gaming abstinence in help-seeking adult problematic gamers. Psychiatry Research. https://doi.org/10.1016/j.psychres.2018.01.008

10. King, D. L., Herd, M. C. E., \& Delfabbro, P. H. (2017). Tolerance in Internet gaming disorder: A need for increasing gaming time or something else? Journal of Behavioral Addictions, 6(4), 525-533. https://doi.org/10.1556/2006.6.2017.072 
11. Ko, C.-H., Lin, H.-C., Lin, P.-C., \& Yen, J.-Y. (2019). Validity, functional impairment and complications related to Internet gaming disorder in the DSM-5 and gaming disorder in the ICD-11. The Australian and New Zealand Journal of Psychiatry, 00(0), 4867419881499. https://doi.org/10.1177/0004867419881499

12. Liang, Q., Yu, C., Chen, Q., Xie, X., Wu, H., Xing, J., ... Dou, K. (2019). Exposure to Community Violence, Affiliations With Risk-Taking Peer Groups, and Internet Gaming Disorder Among Chinese Adolescents: The Moderating Role of Parental Monitoring. Frontiers in Psychology, 10(September). https://doi.org/10.3389/fpsyg.2019.02074

13. Moudiab, S., \& Spada, M. M. (2019). The relative contribution of motives and maladaptive cognitions to levels of Internet Gaming Disorder. Addictive Behaviors Reports, 9(December 2018), 100160. https://doi.org/10.1016/j.abrep.2019.100160

14. Pontes, H. M., Schivinski, B., Brzozowska-woś, M., \& Stavropoulos, V. (2019). Laxer Clinical Criteria for Gaming Disorder May Hinder Future Efforts to Devise an Efficient Diagnostic Approach: A Tree-Based Model Study. Journal of Clinical Medicine, 8(10), 1730. https://doi.org/10.3390/jcm8101730

15. Potenza, M. N., Maurage, P., Brevers, D., Brand, M., \& King, D. L. (2020). Cognitive factors associated with gaming disorder. 221-230. https://doi.org/10.1016/B978-0-12-815298-0.00016-2

16. Quwaider, M., Alabed, A., \& Duwairi, R. (2019). The Impact of Video Games on the Players Behaviors: A Survey. Procedia Computer Science, 151(2018), 575-582. https://doi.org/10.1016/j.procs.2019.04.077

17. Rikkers, W., Lawrence, D., Hafekost, J., \& Zubrick, S. R. (2016). Internet use and electronic gaming by children and adolescents with emotional and behavioural problems in Australia - Results from the second Child and Adolescent Survey of Mental Health and Wellbeing. BMC Public Health, 16(1), 1-17. https://doi.org/10.1186/s12889-016-3058-1

18. Schoenmacker, G. H., Groenman, A. P., Sokolova, E., Oosterlaan, J., Rommelse, N., Roeyers, H., ... Buitelaar, J. K. (2018). Role of conduct problems in the relation between Attention-Deficit Hyperactivity disorder, substance use, and gaming. European Neuropsychopharmacology, 1-12. https://doi.org/10.1016/j.euroneuro.2018.06.003

19. Seok, H. J., Lee, J. M., Park, C., \& Park, J. Y. (2018). Understanding Internet Gaming Addiction among South Korean Adolescents through Photovoice. Children and Youth Services Review, \#pagerange\#. https://doi.org/10.1016/j.childyouth.2018.09.009

20. Stavropoulos, V., Ela, E., Beard, C., Qasim, M., Kuss, D., \& Griffiths, M. (2019). A preliminary cross-cultural study of Hikikomori and Internet Gaming Disorder: The moderating effects of game-playing time and living with parents. Addictive $\begin{array}{llll}\text { Behaviors } \quad \text { Reports, } & \text { 9(September }\end{array}$ https://doi.org/10.1016/j.abrep.2018.10.001

21. Süleyman ÇAKIROĞLU, N. S. (2018). Adaptation of Internet Gaming Disorder Questionnaire to Turkish: Reliability and Validity Study. 30(X), 1-8. 
22. Weinstein, N., Przybylski, A. K., \& Murayama, K. (2017). A prospective study of the motivational and health dynamics of Internet Gaming Disorder. PeerJ, 2017(9). https://doi.org/10.7717/peerj.3838

23. Wu, C. Y., Lee, M. B., Liao, S. C., \& Chang, L. R. (2015). Risk factors of internet addiction among internet users: An online questionnaire survey. PLoS ONE, 10(10), 1-11. https://doi.org/10.1371/journal.pone.0137506

24. World Health Organization. (2018). Gaming Disorder. www.who.int.

25. Young, K. (2009). Understanding Online Gaming Addiction and Treatment Issues for Adolescents. The American Journal of Family Therapy, 37, 355-372. 